\title{
Metabolomics analysis of mangosteen (Garcinia mangostana Linn.) fruit pericarp using different extraction methods and GC-MS
}

\author{
Siti Farah Mamat, Kamalrul Azlan Azizan, Syarul Nataqain Baharum, Normah Mohd Noor, Wan Mohd \\ Aizat*
}

Institute of Systems Biology (INBIOSIS), Universiti Kebangsaan Malaysia (UKM), 43600 Bangi, Selangor, Malaysia

*Corresponding authors: wma@ukm.edu.my

\begin{abstract}
Garcinia mangostana Linn. (mangosteen) reportedly contains several bioactive metabolites which have been used in various traditional disease treatment, nutritional supplement and skincare products. However, reports detailing its characterization and metabolite content are still scarce. Most of the earlier reports only focused on the extraction of specific metabolites such as xanthones and anthocyanins, rather than a comprehensive metabolome profile. In this study, global metabolomics approach using gas chromatography-mass spectrometry (GC-MS) analysis was conducted to profile the metabolite content of mangosteen fruit pericarp harvested at the final stage of ripening (dark purple stage). Five different extraction methods, which vary in their solvent mixtures, solvent ratios, with or without sonication were compared, aimed to maximize metabolite detection from the mangosteen tissue. The results showed that the method using a combined solvent mixture of methanol/chloroform/water (3:1:1 v/v ratio) with sonication has consistent reproducibility amongst biological replicates and successfully yielded the highest number of metabolites compared to the other methods. By combining the results of different extraction methods, we tentatively identified a total of 73 metabolites comprising of sugars (49.32\%), alcohols (9.59\%), sugar acids $(8.22 \%)$, organic acids (6.85\%), phenolic acids (5.48\%), aromatic compounds (2.74\%) and aldehyde (1.37\%). This finding provides an overview of the metabolite content of mangosteen pericarp and comprehensive assessment of extraction methods for an untargeted metabolomics approach of this beneficial fruit.
\end{abstract}

Keywords: fruit; Garcinia mangostana Linn.; GC-MS; metabolite extraction; metabolomics; sample preparation.

Abbreviations: AMDIS_Automated Mass Spectral Deconvolution and Identification System; BSTFA_N,O_bis(trimethylsilyl) trifluoroacetamide; GC-MS_ gas chromatography-mass spectrometry; LC-MS_liquid chromatography-mass spectrometry; MSTFA_N-methyl-N-(trimethylsilyl) trifluoroacetamide; PCA_principal component analysis.

\section{Introduction}

Garcinia mangostana Linn. or commonly known as mangosteen is a climacteric fruit belongs to Clusiaceae family and is a tropical evergreen tree that has been widely planted and grown in southern Vietnam, Myanmar, Thailand and Malaysia. The pericarp of mangosteen is widely used in folk medicines for several purposes particularly for skin infections, wounds, diarrhea, abdominal pain, leucorrhoea, dysentery, suppuration, gonorrhea and chronic ulcer (Shibata et al., 2011; Chaijaroenkul et al., 2014; Mohamed et al., 2014). Mangosteen has been reported to confer various biological and pharmacological benefits including antiinflammation, anti-malaria, anti-allergy, anti-oxidant and anti-cancer (Shibata et al., 2011; Chaijaroenkul et al., 2014; Mohamed et al., 2014; Thong et al., 2015). This is due to the high content of bioactive metabolites such as xanthones, triterpenes, and benzophenones (Nguyen et al., 2005; Mohamed et al., 2014). However, most of the earlier research in mangosteen has mainly focused on xanthones without much emphasis on other potential bioactive metabolites in mangosteen fruit pericarp including primary metabolites (Sukatta et al., 2013; Mishima et al., 2015; Yoswathana and Eshtiaghi, 2015). Hence, further study to profile mangosteen metabolome from the pericarp and optimizing its extraction methods are needed, particularly using the metabolomics approach. Sample preparation including harvesting, drying and extraction is perhaps the most critical step in the experimental design of any metabolomics study (Kim and Verpoorte, 2010). Proper techniques must be applied during this crucial process since it will determine the sensitivity, accuracy and robustness of the whole analysis (Mushtaq et al., 2014; Sapcariu et al., 2014). Metabolite extraction process is one of the most influential process in the metabolomics approach as it will determine how many metabolites and what type of metabolites could be extracted out from any given samples. Before an extraction process is conducted, samples are usually grounded into fine powder to increase their surface area for better absorption of extraction solvent, thus enhancing the release of metabolites from the cells or tissues (Cevallos-Cevallos et al., 2009). There are several factors that should be considered when carrying out the extraction process; these include the polarity and solubility of solvent mixture, temperature, extraction techniques and duration of extraction. For instance, in solvent-based 
extraction, the polarity of solvent determines the amount and types of compounds that could be extracted from samples. Universal polar solvents such as water is excellent in extracting polar metabolites such as sugars, while nonpolar solvents, for example chloroform are suitable for the extraction of non-polar metabolites. However, due to the wide variety of metabolites with different polarities and different concentration levels, at present there is no single solvent that could cover the whole range of metabolites (Kim and Verpoorte, 2010). Amongst the many option of alcohol solvents, methanol and ethanol are often used. The combination of water, methanol and chloroform with varying degree are commonly used in today's metabolomics research. This is to expand the diversity of extracted metabolites, thus maximizing the number of metabolites, covering from non-polar to highly polar, hydrophobic and hydrophilic metabolites (Mushtaq et al., 2014).

Sonication-assisted or microwave-assisted extraction is often conducted to enhance the secretion of metabolites from sample cells or tissues. Sonication techniques applied pulse or high frequency sound waves to disrupt cells, while microwave is used to heat the solvents, commonly exceeding their atmospheric pressure boiling point (Vinatoru, 2001; Kaufmann and Christen, 2002). This suggests that metabolite extraction is one of the most crucial steps to obtain the best metabolite yield in a metabolomics study. Therefore, a metabolite extraction method should be reproducible as well as offering high recovery and stability of the metabolites (De Vos et al., 2007). The last part of sample preparation is the preparation before the analysis. For GC-MS analysis, an additional preparation step which is chemical derivatization is necessary to increase volatility and thermal stability of samples to make them suitable for GC-MS analysis (Kim and Verpoorte, 2010; Okazaki and Saito, 2012). Silylation agents such as $\mathrm{N}$-methyl-N-(trimethylsilyl) trifluoroacetamide (MSTFA) or N,O-bis(trimethylsilyl)trifluoroacetamide (BSTFA) is commonly used for chemical derivatization due to its ion fragmentation stability and provide no interference during GC-MS analysis (Azizan et al., 2012). Furthermore, some samples that are too concentrated might cause poor metabolite spectra formation on the chromatogram, hence it should be diluted before running on either GC-MS, LC-MS or any other type of analysis to obtain clear, sharp and single well separated metabolite peaks.

This study aims to examine different extraction methods and subsequently to maximize the metabolite detection in mangosteen fruit pericarp. Five different extraction methods modified from Lisec et al. (2006), De Vos et al. (2007), a combination method of Lisec et al. (2006) and De Vos et al. (2007), Cadahia et al. (2015) and Okazaki et al. (2016) were compared and evaluated in this study. Specifically, these methods employed different types of solvent mixtures, different solvent ratios and sonication step and thus may lead to a more robust metabolomics analysis. Previously, in most cases, optimization methods of metabolite extraction have only focused on the effects of chemical factors such as different solvent ratios (Zhao et al., 2014) and different solvent mixtures (Dettmer et al., 2011; Theodoridis et al., 2012: Azizan et al., 2015). However, studies conducted to compare both chemical and physical factors simultaneously, as previously performed by Gullberg et al. (2004) for Arabidopsis samples, are still scarce particularly for metabolite-rich samples from fruit. In this study, both chemical (types and ratios of solvents) and physical factors (with or without sonication) were considered for obtaining the most yield using GC-MS analysis. This finding will be useful to provide insights into the metabolite extraction processes and hence allowing better design of metabolite extraction protocols for metabolomics analysis of fruit such as mangosteen.

\section{Results}

\section{Total metabolites in mangosteen pericarp}

Five different extraction methods involving different solvent mixtures, solvent ratios and sonication step were compared and evaluated. Briefly, the metabolite content of mangosteen fruit pericarp was extracted with either method 1 (methanol acidified with formic acid, assisted with sonication), method 2 (methanol/chloroform/water with ratio of 2:1:2), method 3 (methanol/chloroform/water with ratio of $2: 1: 2$, assisted with sonication), method 4 (methanol/chloroform/water with ratio of 3:1:1) or method 5 (methanol/chloroform/water with ratio of 3:1:1, assisted with sonication). In total, 73 metabolites were tentatively identified from all five methods. Method 5 profiled the highest number of total metabolites (34 metabolites), followed by methods 3, 1, 2 and 4 which detected 31, 28, 22 and 15 metabolites, respectively (Table 1). Method 5 also produced the highest number of primary metabolites (28 metabolites), followed by methods $3,2,1$, and 4 which gave 27, 20, 17 and 14 primary metabolites, respectively (Fig 1). For secondary metabolites, method 1 successfully yielded the highest number of secondary metabolites compared to the other extraction methods. Specifically, the predominant peaks belonged to sugar groups, found to be the most abundant metabolites in mangosteen fruit pericarp, covering nearly half of the total metabolites detected (49.32\%) (Fig 1). Second most abundant class of metabolite was alcohols $(9.59 \%)$, followed by sugar acids $(8.22 \%)$, organic acids $(6.85 \%)$ and aldehyde (1.37\%). A small portion of secondary metabolites such as phenolic acids and aromatic compounds (5.48\% and $2.74 \%$, respectively) were also found in the mangosteen fruit pericarp. Meanwhile $16.44 \%$ of the identified peaks belong to metabolites not categorized in any compound chemical classes, thus considered as "others" (Fig 1). Furthermore, $\alpha$-D-glucopyranoside was identified having the highest total peak areas amongst the identified metabolites while D-mannose having the highest total peak areas amongst the metabolites that were present in all extraction methods (Table S1).

\section{Multivariate data analysis}

In this study, unsupervised principal component analysis (PCA) was performed to visualize the grouping patterns and to detect the outliers in the data sets. As shown in Fig 2a, a PCA score plot was obtained from the first two principal components (PCs), PC1 versus PC2, giving the best model with a total variance $\left(R^{2}\right)$ of $53 \%$ and model predictive values $\left(Q^{2}\right)$ of $25 \%$. The metabolites were grouped according to the extraction methods. Method 5 was farther separated from others in the upper right quadrant of the PCA score plot, indicating strong variation in the metabolite concentration 
Table 1. Summary of five different metabolite extraction methods which vary in solvent mixtures, solvent ratio and extraction techniques (sonication). Mangosteen pericarp at the final ripening stage (dark purple colour) was extracted using these methods and analysed using a GC-MS system. The total number of metabolites detected is reported from each method.

\begin{tabular}{|c|c|c|c|c|c|}
\hline $\begin{array}{l}\text { Extraction } \\
\text { method }\end{array}$ & $\begin{array}{l}\text { Original method } \\
\text { references }\end{array}$ & Solvent & Ratio & Sonication & $\begin{array}{c}\text { Number of identified } \\
\text { metabolites }\end{array}$ \\
\hline 1 & De Vos et al. (2007) & $\begin{array}{l}\text { Methanol (75\%) acidified } \\
\text { with } 0.1 \% \text { formic acid }\end{array}$ & 599:1 & Yes & 28 \\
\hline 2 & Lisec et al. (2006) & Methanol: chloroform: water & $2: 1: 2$ & No & 22 \\
\hline 3 & $\begin{array}{c}\text { Lisec et al. (2006) and De } \\
\text { Vos et al. (2007) }\end{array}$ & Methanol: chloroform: water & $2: 1: 2$ & Yes & 31 \\
\hline 4 & Okazaki et al. (2016) & Methanol: chloroform: water & $3: 1: 1$ & No & 15 \\
\hline 5 & Cadahia et al. (2015) & Methanol: chloroform: water & 3:1:1 & Yes & 34 \\
\hline
\end{tabular}

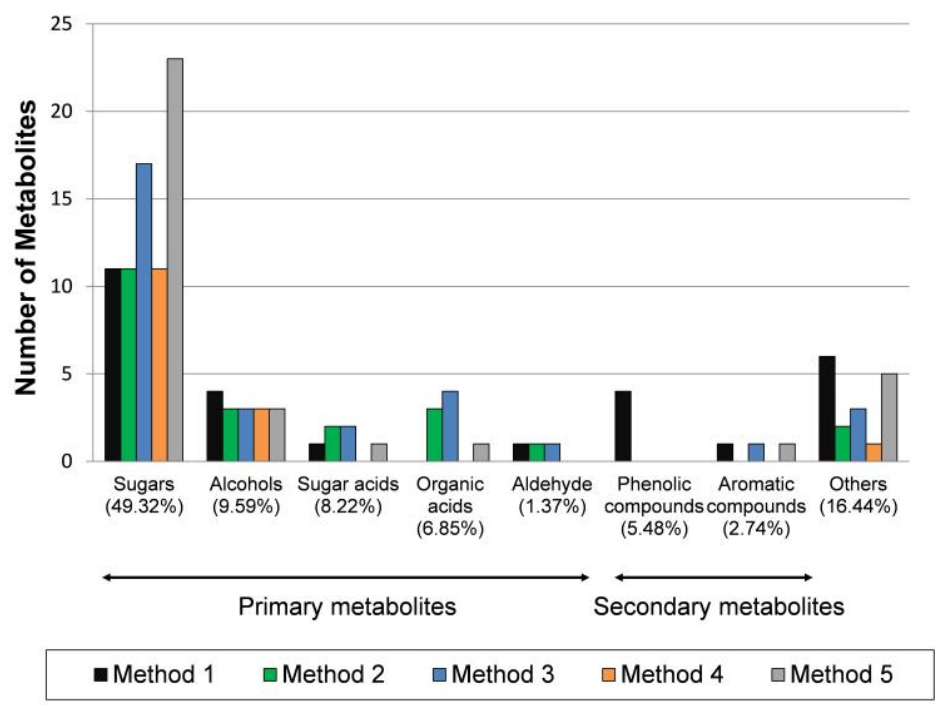

Fig 1. Metabolites detected from five different extraction methods are grouped into different classes of metabolites (primary and secondary metabolites). Each colour represents a different method and the percentage of each chemical class from the total identified metabolites was also presented. Method 1: methanol acidified with formic acid, Method 2: methanol/chloroform/water with ratio of 2:1:2, Method 3: methanol/chloroform/water with ratio of 2:1:2 assisted with sonication, Method 4: methanol/chloroform/water with ratio of 3:1:1 and Method 5: methanol/chloroform/water with ratio of 3:1:1 assisted with sonication.

and composition amongst the extraction method. Methods $1,2,3$, and 4 overlapped in the lower quadrant of the PCA score plot, meaning that there was less variation in the metabolite concentration and composition of these methods. Using a loading plot, metabolites responsible for the separation of method 5 were identified as talose, arabinofuranose, arabinitol, arabinose, malic acids, glucopyranoside and glucopyranose (Fig 2b). Method 2 showed the highest reproducibility as all biological replicates were closed together, compared to the other methods.

\section{Comparison between extraction methods}

Venn diagram demonstrated the similarities and differences between metabolites of the five metabolite extraction methods (Fig 3). Eight metabolites were successfully detected by all five methods including seven primary metabolites (arabinitol, D-glucose, D-ribose, D-mannose, Dfructose, $D$-xylose and D-galactose) and one uncategorized metabolite namely thymol- $\alpha$-d-glucopyranoside (Fig 3, Table S2). Methods 1 and 5 produced the highest number of unique metabolites compared to the other methods, which were 16 and 14 metabolites, respectively (Fig 3). Unique metabolites refer to those metabolites identified only in the extracts from a particular extraction method. Out of the 16 unique metabolites extracted using method 1, five secondary metabolites comprising of four phenolic acids (salicylic acid, benzoic acids, 3,4-dihydroxybenzoic acids and 4-hydroxyphenylethanol) and one aromatic compound (benzaldehyde) were identified (Table S1 and S2). For method 5 , the unique metabolites were mostly sugars such as talose, mannopyranose and D-xylofuranose (Table S2). One sugar acid (pentonic acid) was also detected using this method. Two organic acids, methylmaleic acid and propanedioic acid were recognized as the unique metabolites from method 3 and butanedioic acid as the unique metabolite from method 2. Meanwhile, organic acids such as malic acid and L-(+)-tartaric acid were successfully extracted using more than one extraction methods (malic acid: method 2, 3 and 5; L-(+)-tartaric acid: method 2 and 3). Different sugar acids have also been identified as unique metabolites for different 
a)
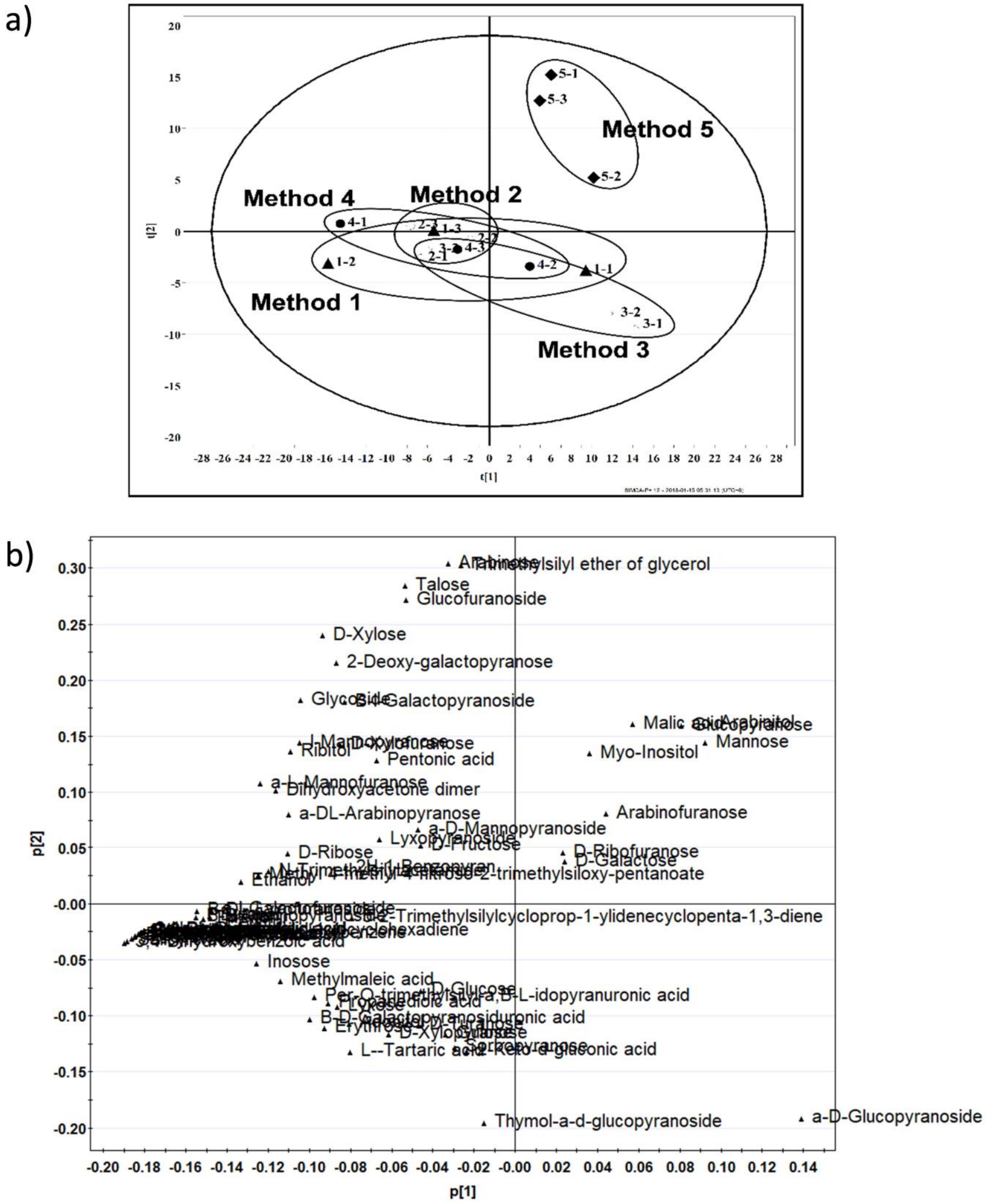

Fig 2. Principal component analysis (PCA) plots for metabolites extracted using five different extraction methods from mangosteen fruit pericarp. Score (a) and loading (b) scatter plots were generated from the first two principle components (PC1 and PC2). Metabolite extraction methods used are as follows; Method 1 (triangle symbols): methanol acidified with formic acid, Method 2 (open circle symbols): methanol/chloroform/water with ratio of 2:1:2, Method 3 (star symbols): methanol/chloroform/water with ratio of 2:1:2 assisted with sonication, Method 4 (closed circle symbols): methanol/chloroform/water with ratio of 3:1:1 and Method 5 (diamond symbols): methanol/chloroform/water with ratio of 3:1:1 assisted with sonication 


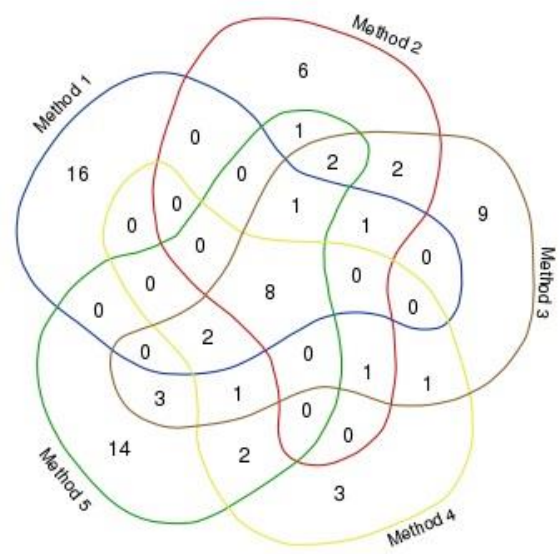

Fig 3. Venn diagram representing the similarities and differences between identified metabolites obtained from the five metabolite extraction methods. Method 1: methanol acidified with formic acid, Method 2: methanol/chloroform/water with ratio of 2:1:2, Method 3: methanol/chloroform/water with ratio of 2:1:2 assisted with sonication, Method 4: methanol/chloroform/water with ratio of 3:1:1 and Method 5: methanol/chloroform/water with ratio of 3:1:1 assisted with sonication.

metabolite extraction methods. For instance, ribonic acid and mannonic acid were only present in mangosteen extract from method 2, while $\beta$-D-galactopyranosiduronic acid and 2-keto-d-gluconic acid were only detected using method 3. Other sugar acids, gluconic acid and pentonic acid were the unique metabolites from method 1 and method 5, respectively (Table S2).

\section{Discussion}

\section{Total metabolites}

Combination of different solvents showed improvement in the detection of some chemical classes of metabolites. The combination of methanol, chloroform and water showed an excellent extraction results, covering different metabolites classes including sugars, alcohols, organic acids and also secondary metabolites (Fig 1). This is because the mixture preferentially extracts both polar and non-polar metabolites from any given plant samples, hence increasing the coverage of metabolome profiles. Extraction methods with sonication gave higher numbers of total metabolites compared to those methods without sonication (Table 1). This suggests that the sonication process enhanced the release of metabolites from the mangosteen pericarp regardless of extraction solvents used and hence should be adopted in any metabolite extraction method. Sonication has also been proven to increase extracted metabolites from various other sample tissues, for examples herbs (Vinatoru, 2001), soybean (Rostagno et al., 2003) and human endometrial carcinoma (ECC1) cell lines (Matheus et al., 2014).

\section{Primary metabolites}

The metabolites successfully detected by GC-MS analysis comprised of mainly primary metabolites, especially sugars (Fig 1). Primary metabolites are central to life processes and play key roles for plant growth, development and survival (Salunke et al., 2009; Kumar et al., 2014).

Since the mangosteen fruit was harvested at the final stage of ripening (dark purple stage), the sugar content was expected to be higher compared to the other metabolites.
This is due to the biochemical changes in fruit tissues including pericarp that lead to carbohydrate breakdown and the accumulation of sugars as a result of increased gluconeogenesis during ripening process (Othman, 2012; Osorio et al., 2013; Aizat et al., 2014). Alcohols, including sugar alcohols as well as sugar acids and organic acids were also identified but at lower percentages (approximately 6$10 \%)$ compared to sugars (49.32\%) (Fig 1). The levels of sugars and organic acids can contribute to the combination of sweetness and acidity of fruit during ripening process (Lee et al., 2015). However, as this study only used the fully ripened mangosteen fruit pericarp, a more thorough study using different ripening stages and tissues (pericarp and aril) is needed to elucidate sugar and organic acid changes throughout its ripening process.

\section{Secondary metabolites}

Secondary metabolites are organic molecules that derived from primary metabolites, responsible for plant defense mechanism against various factors such as pests, environmental conditions and diseases (Salunke et al., 2009; Kumar et al., 2014). Six secondary metabolites were found in mangosteen fruit pericarp comprising of four phenolic compounds (salicylic acid, benzoic acids, 3,4dihydroxybenzoic acid and 4-hydroxyphenylethanol) and two aromatic compounds (2H-1-benzopyran and benzaldehyde) (Table S1). Salicylic acid is a plant immune signal produced upon pathogen attack (Fu et al., 2012) and believed to play significant roles in plant defense mechanism (Senaratna et al., 2000; Zhang et al., 2003; Clarke et al., 2004). The hormone is also reported to influence various plant mechanisms directly or indirectly particularly in plant growth and development, respiration, senescenceassociated gene expression, fruit yield and enzyme activities (Vlot et al., 2009; Yusuf et al., 2013). Benzoic acid and its derivative, 3,4-dihydroxybenzoic acid (also known as protocatechuic acid) are natural phenolic acids that have been reported to possess several pharmacological benefits including anti-coagulatory, anti-oxidative, and antiinflammatory (Lin et al., 2009) and anti-cancer (Hudson et al., 2000; Tanaka et al., 2011). 
Previous study by Zadernowski et al. (2009) on phenolic acids profiling of mangosteen revealed that 3,4dihydroxybenzoic was the major phenolic acids in mangosteen fruit pericarp. The same pattern could be observed in this study where 3,4-dihydroxybenzoic possesses the major peak area compared to other phenolic acids identified (Table S1). This metabolite is also widely distributed in medicinal plants and other fruit such as rooibos tea (Aspalathus linearis) (von Gadow et al., 1997), brown rice (Oryza sativa L.) (Hudson et al., 2000), prune (Prunus domestica L.) (Kayano et al., 2002), almond (Prunus amygdalus Batsch) (Sang et al., 2002) and also in Chinese herbal medicines (Li et al., 2011). Meanwhile, another phenolic compound detected was 4-hydroxyphenylethanol or commonly known as tyrosol. This compound has been reported to be the main biophenol for the oil, leaves, stem and roots of olive plant that exert antioxidant and radical scavenger activities ( Del Río et al., 2003; Di Benedetto et al., 2007; Ortega-García and Peragón). Interestingly, a study by Ortega-García and Peragón (2010) revealed that the concentration of tyrosol changes significantly at the root and stem part of olive tree during ripening. This metabolite might also contribute to the antioxidant activities in mangosteen fruit pericarp and perhaps involved in the metabolism of this fruit throughout the ripening process. As for the aromatic compounds, they are believed to provide characteristic aromas to ripening fruit, making it attractive towards seeds dispersal agents and consumers (Osorio et al., 2013; Lee et al., 2015). In this study, two aromatic compounds were successfully detected which are $2 \mathrm{H}-1$ benzopyran and benzaldehyde.

Previously, the combination of methanol and formic acid used in method 1 was suggested to be a suitable solvent for an efficient extraction of a wide range of metabolites, covering various secondary metabolite from tissues and plant species (De Vos et al., 2007). However, in this study, the total number of secondary metabolites detected was relatively small. This may be due to the limitation of GC-MS which mostly detects volatile or easily volatilized metabolites such as sugars. Generally, GC-MS analysis is mainly used to detect primary metabolites such as sugars, alcohols, organic acids and amino acids (Gullberg et al., 2004; t'Kindt et al., 2009; Lee et al., 2013; Okazaki et al., 2016;) whereas LC-MS is more suitable for the detection of secondary metabolites such as flavonoids, alkaloids, saponins and phenylpropanoids (De Vos et al., 2007; t'Kindt et al., 2009; Lee et al., 2013). The future use of LC-MS system may improve the detection of secondary metabolites and hence the profiling of the mangosteen metabolome.

\section{Materials and Methods}

\section{Chemicals \& reagents}

Analytical grade solvents such as methanol and chloroform were purchased from Merck (Germany). Methoxyamine hydrochloride (MeOX) in pyridine and MSTFA were purchased from Sigma Aldrich (St. Loius, MO, USA).

\section{Plant materials}

Mangosteen fruit were collected from Universiti Kebangsaan Malaysia (UKM) experimental plots, located at Bangi,
Selangor, Malaysia $\left(2^{\circ} 55^{\prime} 09.0^{\prime \prime} \mathrm{N} \quad 101^{\circ} 47^{\prime} 04.8^{\prime \prime} \mathrm{E}\right)$ during mangosteen seasonal period in June-August, 2014. Fruit were harvested once they reached dark purple stage which is the last stage of mangosteen ripening (Osman and Milan, 2006). Mangosteen fruit pericarp were then separated from other tissues and ground into fine powder using laboratorygrade blender before dried using freeze-dryer to remove the water content. Samples were kept in $-80^{\circ} \mathrm{C}$ prior to extraction. Three biological replicates (fruit) of mangosteen pericarp were prepared for each metabolite extraction method.

\section{Metabolite extraction}

\section{Method 1}

Method 1 was obtained from De Vos et al. (2007) with slight modifications. Approximately $200 \mathrm{mg}$ of freeze-dried sample powder was mixed with freshly prepared ice-cold extraction solution $(599 \mu \mathrm{L}$ of $75 \%$ methanol acidified with $1.0 \mu \mathrm{L}$ of $0.1 \%$ formic acid) in a volume per fresh weight ratio of three to one. The mixture was immediately vortexed for 10 seconds. The mixture was then sonicated for 20 minutes at maximum frequency $(40 \mathrm{kHz})$ continuously, in a water bath at room temperature. After that, the mixture was centrifuged for 10 minutes at maximum speed $(16,100 \times \mathrm{g})$ at room temperature and the supernatant was collected in a fresh tube leaving the pellet out.

\section{Method 2}

Method 2 was obtained from Lisec et al. (2006) with slight modifications. Approximately $200 \mathrm{mg}$ of freeze-dried sample was homogenized with $1,500 \mu \mathrm{l}$ of ice-cold $100 \%$ methanol and vortexed for 10 seconds. The mixture was incubated for 10 minutes in a water bath at $70^{\circ} \mathrm{C}$. Then, the mixture was centrifuged for 10 minutes at $11,000 \times g$ and the supernatant obtained was transferred to a new tube. After that, $750 \mu \mathrm{l}$ of $100 \%$ ice-cold chloroform was added followed by $1,500 \mu \mathrm{ld} \mathrm{dH}_{2} \mathrm{O}$. Then, the mixture was vortexed for 10 seconds and then centrifuged for 15 minutes at 2,200 $\times \mathrm{g}$. Lastly, the supernatant from the upper phase (polar phase) was aliquoted into a fresh $1.5-\mathrm{ml}$ tube.

\section{Method 3}

Method 3 was the modified method based on method 1 and method 2. This was done to evaluate the efficiency of sonication-assisted steps employed in method 1 using the extraction solvent from method 2. Approximately $200 \mathrm{mg}$ of frozen sample powder was mixed with ice-cold sample extraction solution containing $1,500 \mu \mathrm{l}$ of $100 \%$ methanol, $750 \mu \mathrm{l}$ of $100 \%$ chloroform and $1,500 \mu \mathrm{l}$ of $\mathrm{H}_{2} \mathrm{O}$ (Lisec et. al 2006). The mixture was vortexed (immediately) for 10 seconds and sonicated for 15 minutes at maximum frequency $(40 \mathrm{kHz})$ continuously, in a water bath at room temperature. Then, the mixture was centrifuged for 10 minutes at maximum speed $(16,100 \times g)$ at room temperature. The supernatant was collected in a new tube.

\section{Method 4}

Method 4 was obtained from Okazaki et al. (2016) with slight modifications. Approximately $1500 \mu \mathrm{l}$ of a $(3: 1: 1 \mathrm{v} / \mathrm{v})$ 
mixture of ice-cold extraction medium methanol/ chloroform/ water (100\% concentration each) was mixed with $200 \mathrm{mg}$ of frozen sample powder. Then, the sample was vortexed for 30 seconds and left on ice for 30 minutes before $400 \mu \mathrm{l}$ of distilled water was added (to extract the aqueous metabolite). The extract was vortexed again for 30 seconds. After that, the mixture was centrifuged at 1,400 $\mathrm{rpm}$ at $4^{\circ} \mathrm{C}$ for two minutes to separate their layers. The supernatant obtained was transferred into a new pre-cooled Eppendorf tube.

\section{Method 5}

Method 5 was conducted based on Cahadia et al. (2015) with slight modifications. Approximately $200 \mathrm{mg}$ of sample powder was mixed with $400 \mu \mathrm{L}$ of $100 \%$ ice-cold chloroform and sonicated in an ultrasonication bath for 20 minutes at room temperature. Then, $1,600 \mu \mathrm{L}$ of ice-cold water and $100 \%$ methanol $(1: 3 \mathrm{v} / \mathrm{v})$ was added and the mixture was sonicated again for 20 minutes. The mixture was centrifuged at maximum speed $(16,100 \times \mathrm{g})$ for 10 minutes and then the supernatant was extracted.

\section{Sample derivatization}

Approximately $50 \mu$ l of the supernatant from each extraction method was further derivatized. Firstly, the supernatant was dried in a vacuum concentrator. The dried supernatant was then dissolved with $40 \mu \mathrm{l}$ of $20 \mathrm{mg} / \mathrm{ml} \mathrm{MeOX}$ in pyridine and incubated at $40^{\circ} \mathrm{C}$ for 90 minutes. Then, $40 \mu \mathrm{l}$ MSTFA was added and the mixture was incubated again at $40^{\circ} \mathrm{C}$ for 30 minutes.

\section{GC-MS parameter}

Sample was analyzed using Perkin Elmer Clarus 600 Turbo Mass GC-MS (Perkin Elmer, USA) coupled to quadrupole type MS operated at $70 \mathrm{eV}$. An aliquout of $1.0 \mu \mathrm{l}$ sample was injected into Elite 5MS (5\% diphenyl 95\% dimethylpolysiloxane, $30.0 \mathrm{~m} \times 0.25 \mathrm{~mm}$ ID $\times 250 \mu \mathrm{m}$ ) column. Helium was used as a carrier gas and the scan range was set to 50-600 Da. The initial oven temperature was set to $70^{\circ} \mathrm{C}$ for $1 \mathrm{~min}$ and was increased by $1^{\circ} \mathrm{C} / \mathrm{min}$ to $76^{\circ} \mathrm{C}$, then held for $1 \mathrm{~min}$ and increased by $6^{\circ} \mathrm{C} / \mathrm{min}$ to $300^{\circ} \mathrm{C}$ and finally held for 5 minutes. Both injector and transfer temperatures were set to $250^{\circ} \mathrm{C}$ while the source temperature was adjusted to $300^{\circ} \mathrm{C}$. The full scan range was acquired after 8 minutes (solvent delay) with the split ratio of $50: 1$.

\section{Data processing}

Raw GC-MS data table with metabolite name, retention time, match, relative match and peak area was generated using Turbo Mass software (Perkin Elmer, USA). Identification was carried out using NIST (National Institute of Standards and Technology) mass spectral library (2005) with match cut off of 700 . The raw GC-MS data was then imported to AMDIS (Automated Mass Spectral Deconvolution and Identification System) software for further identification of overlapped peaks and to retrieve individual mass spectra. The extracted output data from AMDIS was aligned and annotated before imported to
MetaboAnalyst 3.0 server (www.metaboanalyst.ca) for further integral normalization. In MetaboAnalyst, the data was normalized by the highest total peak area (D-mannose) and subjected to log transformation (Xia et al. 2015). Normalized and validated data table was exported to SIMCA$\mathrm{P}^{+}$version 12 (Umetrics $\mathrm{AB}$, Umea, Sweden) for multivariate analysis.

\section{Conclusion}

Metabolomics approach utilizing GC-MS and different extraction methods was used to profile the metabolite content of mangosteen fruit pericarp at the latest stage of ripening. The combination of different solvent mixtures, solvent ratios and sonication influenced the overall efficiency, accuracy and robustness of metabolomics analysis. Metabolite extraction method using a solvent mixture of methanol, chloroform and water with a ratio of 3:1:1 assisted with sonication gave the highest yield of total metabolites and primary metabolites. Meanwhile, a solvent mixture of methanol and formic acids gave the highest yield of secondary metabolites compared to other methods measured in this study. The findings could be useful for further optimization of metabolite extraction method for various purposes specifically to identify and characterize the metabolite composition in mangosteen fruit pericarp or any other plant extracts.

\section{Acknowledgements}

This research was funded by the Sciencefund grant (02-0102-SF1237) from the Malaysian Ministry of Science, Technology and Innovation (MOSTI), Geran Galakan Penyelidik Muda (GGPM-2014-008) from Universiti Kebangsaan Malaysia (UKM) and Fundamental Research Grant Scheme (FRGS/2/2014/SG05/UKM/02/2) from the Malaysian Ministry of Higher Education (MOHE).

\section{References}

Aizat WM, Dias DA, Stangoulis JC, Able JA, Roessner U, Able AJ (2014) Metabolomics of capsicum ripening reveals modification of the ethylene related-pathway and carbon metabolism. Postharvest Biol Technol. 89:19-31.

Azizan KA, Baharum SN, Ressom HW, Noor NM (2012) GCMS analysis and PLS-DA validation of the trimethylsilylderivatization techniques. Am J Appl Sci. 9:1124-1136.

Azizan KA, Ghani NHA, Nawawi MF (2015) GC-MS based metabolomics and multivariate statistical analysis of Wedelia trilobata extracts for the identification of potential phytochemical properties. Plant Omics. 8:537543.

Cadahia E, Fernandez De Simon B, Aranda I, Sanz M, Sanchez-Gomez D, Pinto E (2015) Non-targeted metabolomic profile of Fagus sylvatica L. leaves using liquid chromatography with mass spectrometry and gas chromatography with mass spectrometry. Phytochem Analysis. 26:171-182.

Cevallos-Cevallos JM, Reyes-De-Corcuera JI, Etxeberria E, Danyluk MD, Rodrick GE (2009) Metabolomic analysis in food science: a review. Trends Food Sci Tech. 20:557-566. 
Chaijaroenkul W, Mubaraki MA, Ward SA, Na-Bangchang K (2014) Metabolite footprinting of Plasmodium falciparum following exposure to Garcinia mangostana Linn. crude extract. Exp Parasitol. 145: 80-86.

Clarke SM, Mur LA, Wood JE, Scott IM (2004) Salicylic acid dependent signaling promotes basal thermotolerance but is not essential for acquired thermotolerance in Arabidopsis thaliana. Plant J. 38:432-447.

De Vos RC, Moco S, Lommen A, Keurentjes JJ, Bino RJ, Hall RD (2007) Untargeted large scale plant metabolomics using liquid chromatography coupled to mass spectrometry. Nat Protoc. 2:778-791.

Del Río J, Báidez A, Botía JM, Ortuno A (2003) Enhancement of phenolic compounds in olive plants (Olea europaea L.) and their influence on resistance against Phytophthora sp. Food Chem. 83:75-78.

Dettmer K, Nürnberger N, Kaspar H, Gruber MA, Almstetter MF, Oefner PJ (2011) Metabolite extraction from adherently growing mammalian cells for metabolomics studies: optimization of harvesting and extraction protocols. Anal Bioanal Chem. 399:1127-1139.

Di Benedetto R, Varì R, Scazzocchio B, Filesi C, Santangelo C, Giovannini C, Matarrese P, D'archivio M, Masella R (2007) Tyrosol, the major extra virgin olive oil compound, restored intracellular antioxidant defences in spite of its weak antioxidative effectiveness. Nutr Metab Cardiovas. 17:535-545.

Fu ZQ, Yan S, Saleh A, Wang W, Ruble J, Oka N, Mohan R, Spoel SH, Tada Y, Zheng N (2012) NPR3 and NPR4 are receptors for the immune signal salicylic acid in plants. Nature. 486:228-232.

Gullberg J, Jonsson P, Nordström A, Sjöström M, Moritz T, Moritz T (2004) Design of experiments: an efficient strategy to identify factors influencing extraction and derivatization of Arabidopsis thaliana samples in metabolomic studies with gas chromatography/mass spectrometry. Anal Biochem. 331:283-295.

Hudson EA, Dinh PA, Kokubun T, Simmonds MS, Gescher A (2000) Characterization of potentially chemopreventive phenols in extracts of brown rice that inhibit the growth of human breast and colon cancer cells. Cancer Epidem Biomar. 9:1163-1170.

Kaufmann B, Christen P (2002) Recent extraction techniques for natural products: microwave-assisted extraction and pressurised solvent extraction. Phytochem Analysis. 13:105-113.

Kayano S-I, Kikuzaki H, Fukutsuka N, Mitani T, Nakatani N (2002) Antioxidant activity ofprune (Prunus domestica L.) constituents and a new synergist. J Agr Food Chem. 50:3708-3712.

Kim HK, Verpoorte R (2010) Sample preparation for plant metabolomics. Phytochem Analysis. 21:4-13.

Kumar A, Yadav A, Gupta N, Kumar S, Yadav V, Prakash A, Gurjar H (2014) Metabolites in plants and its classification. World J Pharm Pharm Sci. 4:287-305.

Lee $H J$, Suh DH, Jung ES, Park HM, Jung G-Y, Do S-G, Lee $\mathrm{CH}$ (2015) Metabolomics of Lonicera caerulea fruit during ripening and its relationship with color and antioxidant activity. Food Res Int. 78:343-351.

Li X, Wang X, Chen D, Chen S (2011) Antioxidant activity and mechanism of protocatechuic acid in vitro. Funct Food Health Dis. 1:232-244.
Lin C-Y, Huang C-S, Huang C-Y, Yin M-C (2009) Anticoagulatory, antiinflammatory, and antioxidative effects of protocatechuic acid in diabetic mice. J Agr Food Chem. 57:6661-6667.

Lisec J, Schauer N, Kopka J, Willmitzer L, Fernie AR (2006) Gas chromatography mass spectrometry-based metabolite profiling in plants. Nat Protoc. 1:387.

Matheus N, Hansen S, Rozet E, Peixoto P, Maquoi E, Lambert $V$, Noël A, Frédérich $M$, Mottet $D$, Tullio $P$ (2014) An easy, convenient cell and tissue extraction protocol

for nuclear magnetic resonance metabolomics. Phytochem Analysis. 25:342-349.

Mishima K, Kawamura $\mathrm{H}$, Ito $\mathrm{S}$, Inoue $\mathrm{Y}$, Hirota $\mathrm{T}$, Kato $\mathrm{T}$, Sharmin T, Kawakami R, Irie K, Mishima K (2015) Liquid carbon dioxide extraction of xanthones from the pericarps of Garcinia mangostana Linn. enhanced by ultrasonic irradiation. Solvent Extr Res Dev. 22:187-199.

Mohamed GA, Ibrahim SR, Shaaban MI, Ross SA (2014) Mangostana xanthones I and II, new xanthones from the pericarp of Garcinia mangostana. Fitoterapia. 98:215-221.

Mushtaq MY, Choi YH, Verpoorte R, Wilson EG (2014) Extraction for metabolomics: access to the metabolome. Phytochem Analysis. 25:291-306.

Nguyen L-HD, Venkatraman G, Sim K-Y, Harrison U (2005) Xanthones and benzophenones from Garcinia griffithii and Garcinia mangostana. Phytochemistry. 66:1718-1723.

Okazaki K, Kimura Y, Sugiyama K, Kami D, Nakamura T, Oka $N$ (2016) Discovering metabolic indices for early detection of squash (Cucurbita maxima) storage quality using GCMS-based metabolite profiling. Food Chem. 196:11501155.

Okazaki Y, Saito K (2012) Recent advances of metabolomics in plant biotechnology. Plant Biotechnol Rep. 6:1-15.

Ortega-García F, Peragón J (2010) HPLC analysis of oleuropein, hydroxytyrosol, and tyrosol in stems and roots of Olea europaea L. cv. Picual during ripening. J Sci Food Agr. 90:2295-2300.

Osman MB, Milan AR (2006) Fruits for the future 9: Mangosteen Garcinia mangostana. Southampton Centre for Underutilised Crops, University of Southampton, Southampton, United Kingdom.

Osorio S, Scossa F, Fernie A (2013) Molecular regulation of fruit ripening. Front Plant Sci. 4:198.

Othman BA (2012) Diverse mechanisms of pectic polysaccharide degradation distinguished in fruit cell walls in vivo. Dissertation, The University of Edinburgh, Scotland.

Rostagno MA, Palma M, Barroso CG (2003) Ultrasoundassisted extraction of soy isoflavones. J Chromatogr A. 1012:119-128

Salunke B, Prakash K, Vishwakarma K, Maheshwari V (2009) Plant metabolites: an alternative and sustainable approach towards post harvest pest management in pulses. Physiol Mol Biol Pla. 15:185-197.

Sang S, Lapsley K, Jeong W-S, Lachance PA, Ho C-T, Rosen RT (2002) Antioxidative phenolic compounds isolated from almond skins (Prunus amygdalus Batsch). J Agr Food Chem. 50:2459-2463.

Sapcariu SC, Kanashova T, Weindl D, Ghelfi J, Dittmar G, Hiller K (2014) Simultaneous extraction of proteins and metabolites from cells in culture. MethodsX. 1:74-80. 
Senaratna T, Touchell D, Bunn E, Dixon K (2000) Acetyl salicylic acid (aspirin) and salicylic acid induce multiple stress tolerance in bean and tomato plants. Plant Growth Regul. 30:157-161.

Shibata $M-A$, linuma $M$, Morimoto J, Kurose $H$, Akamatsu $K$, Okuno Y, Akao Y, Otsuki Y (2011) $\alpha$-Mangostin extracted from the pericarp of the mangosteen (Garcinia mangostana Linn.) reduces tumor growth and lymph node metastasis in an immunocompetent xenograft model of metastatic mammary cancer carrying a p53 mutation. BMC Med. 9:69.

Sukatta U, Takenaka M, Ono H, Okadome H, Sotome I, Nanayama K, Thanapase W, Isobe S (2013) Distribution of major xanthones in the pericarp, aril, and yellow gum of mangosteen (Garcinia mangostana Linn.) fruit and their contribution to antioxidative activity. Biosci Biotech Bioch. 77:984-987.

Tanaka T, Tanaka T, Tanaka M (2011) Potential cancer chemopreventive activity of protocatechuic acid. J Exp Clin Med. 3:27-33.

Theodoridis G, Gika H, Franceschi P, Caputi L, Arapitsas P, Scholz M, Masuero D, Wehrens R, Vrhovsek U, Mattivi F (2012) LC-MS based global metabolite profiling of grapes: solvent extraction protocol optimisation. Metabolomics. 8:175-185.

Thong NM, Quang DT, Bui NHT, Dao DQ, Nam PC (2015) Antioxidant properties of xanthones extracted from the pericarp of Garcinia mangostana (Mangosteen): A theoretical study. Chem Phys Lett. 625:30-35.

t'Kindt R, Morreel K, Deforce D, Boerjan W, Van Bocxlaer J (2009) Joint GC-MS and LC-MS platforms for comprehensive plant metabolomics: Repeatability and sample pre-treatment. J Chromatogr B. 877:3572-3580.
Vinatoru M (2001) An overview of the ultrasonically assisted extraction of bioactive principles from herbs. Ultrason Sonochem. 8:303-313.

Vlot AC, Dempsey DMA, Klessig DF (2009) Salicylic acid, a multifaceted hormone to combat disease. Annu Rev Phytopathol. 47:177-206.

von Gadow A, Joubert E, Hansmann CF (1997) Comparison of the antioxidant activity of aspalathin with that of other plant phenols of rooibos tea (Aspalathus linearis), $\alpha$ tocopherol, BHT, and BHA. J Agr Food Chem. 45:632-638.

Xia J, Sinelnikov IV, Han B, Wishart DS (2015) MetaboAnalyst 3.0-making metabolomics more meaningful. Nucleic Acids Res. 43:W251-W257.

Yoswathana N, Eshtiaghi M (2015) Optimization of subcritical ethanol extraction for xanthone from mangosteen pericarp. Int J Chem Eng Appl. 6:115.

Yusuf $M$, Hayat S, Alyemeni MN, Fariduddin $Q$, Ahmad A (2013) Salicylic acid: physiological roles in plants. In: Hayat S, Ahmad A, Alyemeni M (eds) Salicylic acid. Springer, Dordrecht.

Zadernowski R, Czaplicki S, Naczk M (2009) Phenolic acid profiles of mangosteen fruits (Garcinia mangostana). Food Chem. 112:685-689.

Zhang Y, Chen K, Zhang S, Ferguson I (2003) The role of salicylic acid in postharvest ripening of kiwifruit. Postharvest Biol Tec. 28:67-74.

Zhao J, Hu C, Zeng J, Zhao Y, Zhang J, Chang Y, Li L, Zhao C, Lu $X, X u$ G (2014) Study of polar metabolites in tobacco from different geographical origins by using capillary electrophoresis-mass spectrometry. Metabolomics. 10:805-815. 